


\title{
An Autonomous Programmable Actuator and Shape Reconfigurable Structures Using Bistability and Shape Memory Polymers
}

\author{
Tian Chen and Kristina Shea
}

\begin{abstract}
Autonomous deployment and shape reconfiguration of structures are a crucial field of research in space exploration with emerging applications in the automotive, building, and biomedical industries. Challenges in achieving autonomy include the following: bulky energy sources, imprecise deployment, jamming of components, and lack of structural integrity. Leveraging advances in the fields of shape memory polymers, bistability, and three-dimensional (3D) multimaterial printing, we present a 3D-printed programmable actuator that enables the autonomous deployment and shape reconfiguration of structures activated through surrounding temperature change. Using a shape memory polymer as the temperature controllable energy source and a bistable mechanism as the linear actuator and force amplifier, the structures achieve precise geometric activation and quantifiable load-bearing capacity. The proposed unit actuator integrates these two components and is designed to be assembled into larger deployable and shape reconfigurable structures. First, we demonstrate that the activation of the unit actuator can be sequenced by tailoring each shape memory polymer to a different activation time. Next, by changing the configuration of the actuator, we demonstrate an initially flat surface that transforms into a pyramid or a hyperbolic paraboloid, thus demonstrating a multistate structure. Load-bearing capability is demonstrated for both during activation and in the operating state.
\end{abstract}

Keywords: reconfigurable structures, programmable matter, bistability, shape memory polymer, 4D printing

\section{Introduction}

DEPLOYABLE STRUCTURES ARE used in a wide range of applications in space exploration, ${ }^{1}$ biomedical, ${ }^{2}$ and solar energy. ${ }^{3}$ In these applications, the properties of the deployable structures typically sought include large-scale shape change, predictable geometric transformations and reconfigurations, passively controllable activation, load-bearing capability, and tunable deployment conditions. In addition to motorized actuators, a number of passively actuated designs have been proposed, including electricity, ${ }^{4}$ light, ${ }^{5}$ pressure, ${ }^{6}$ shape memory effect (SME), ${ }^{7}$ swelling, ${ }^{8}$ and piezoelectricity. ${ }^{9}$
In the field of space exploration, deployment is a critical phase in the life cycle of a space structure. ${ }^{10}$ With respect to kinematics, entanglement and jamming may occur if part movements cannot be precisely controlled. ${ }^{11}$ Mechanically, the driving force must be tuned to overcome resistance at all stages of deployment. ${ }^{12}$ Traditionally, designs often involve complex packing strategies, such as origami folding, ${ }^{13}$ tensegrity, ${ }^{14}$ as well as bulky powered driving mechanisms such as air pressure ${ }^{15}$ and electrical drives. ${ }^{16}$ With multimaterial three-dimensional (3D) printing technologies, there is potential to fabricate monolithic designs that can be autonomously activated by environmental triggers, for example, temperature.

Engineering Design and Computing Laboratory, Department of Mechanical and Process Engineering, Swiss Federal Institute of Technology Zurich, Zurich, Switzerland.

(C) Tian Chen and Kristina Shea 2018; Published by Mary Ann Liebert, Inc. This Open Access article is distributed under the terms of the Creative Commons License (http://creativecommons.org/licenses/by/4.0), which permits unrestricted use, distribution, and reproduction in any medium, provided the original work is properly cited.

Opposite page: A flat structure is activated through temperature change to autonomously create two different load bearing structures, thus demonstrating a multistate, reconfigurable structure. Photo credit: Jung-Chew Tse, Tian Chen (EDAC, D-MAVT, ETH Zurich) 
An increasing number of active structures are designed and fabricated in a newly coined field of four-dimensional (4D) printing. ${ }^{8} 4 \mathrm{D}$ printing uses properties of $3 \mathrm{D}$ printing materials to achieve design shape change under environmental forces. The development has been focused in the areas of material synthesis and geometric reconfiguration. In Raviv et al. ${ }^{17} \mathrm{a}$ hydrogel that swells under water is used as an actuator that changes the shape of a compliant string or surface. Sydney Gladman et al. ${ }^{18}$ continued this method of actuation by designing anisotropic properties of a swelling meta-material. Ge et $a l .{ }^{19}$ first utilized the shape memory properties of the photopolymeric inks used in the Polyjet 3D printing technology to achieve shape change.

Each of these actuation methods has areas for improvement. Swelling in a hydrogel has a long activation time, imprecise geometric configuration change, and lack of loadbearing capacity. In addition, it is often impractical to submerge a structure under water. Shape memory polymers, as implemented by Ge et al. ${ }^{19}$ require the design to be fabricated in the activated state, while offering no real precision in the programmed state.

The SME of polymers is adopted in this research as the activation material since it is able to produce a large actuation strain $^{20}$ and has a moderate recovery stress. While the SME is observed with most thermosets, it is not feasible for traditional fabrication methods to produce complex parts with multiple materials. In this work, we exploit the multimaterial inkjet technology to fabricate complex designs by selectively depositing photoreactive liquid resins of different stiffness and glass transition temperatures. Currently, there is no other means of fabrication to do this.

By combining this polymer with a bistable mechanism, called a von Mises truss, we are able to define two distinct equilibrium states that can be precisely achieved. Von Mises type bistable mechanisms have previously been used in active masts, ${ }^{21}$ for energy absorption, ${ }^{22}$ and more recently to create $3 \mathrm{D}$-printed reconfigurable structures. ${ }^{23}$

In the activation phase, the bistable mechanism acts as a force amplifier and renders the deployable structure load bearing. By combining the mechanics of bistability and shape memory polymers, we present a unit actuator that delivers the above characteristics and addresses the shortcomings. We propose to integrate this actuator in deployable structures as the source of activation for shape reconfiguration. The structures can be deployed from two-dimensional (2D) to 3D states when the temperature reaches a threshold. By demonstrating a synclastic and an anticlastic structure reconfigured from the same base design, we demonstrate both autonomous deployment and shape reconfiguration.

While it is possible to control individual shape memory elements through Joule heating, one of the aims of this work is to eliminate onboard electrical components in the proposed structures. Instead, activation is controlled through the surrounding environment. In particular, we activate the struc- tures using heated water to simulate a reduced gravity environment. This is typically used to test space deploying structures and machineries ${ }^{24}$ and provides more uniform heating compared to say a heat lamp. A similar application on a microscopic level would be the activation of microstructures in blood vessels through body heat.

\section{Design and Results}

\section{Design of a programmable unit actuator using bistability and shape memory polymers}

First, we introduce the geometry of the actuator design, second, we discuss the kinematics and activation sequence, and finally, we investigate its mechanical behavior. For actuation, we propose a unit actuator consisting of a bistable mechanism that dictates the equilibrium states and shape change and the shape memory strip (SMS) that provides the activation force. This unit actuator is capable of activating independently of the rest of the structure. The principle behind this proposed actuator is shown in Figure 1a, where the SME is used to trigger bistability from the contracted to the expanded configuration or vice versa.

The bistable mechanism is a realization of the von Mises truss $^{25}$ and is adapted from designs proposed by Chen et al. ${ }^{23}$ It is fabricated using the rigid truss members, the compliant joint and beam. It has been shown that the bistable force can be increased by increasing the joint material stiffness or decreasing the joint length. The flexible beam is added to better simulate the ideal boundary condition (Fig. 1b). The design is fabricated in either the contracted or the expanded configuration and is triggered by the SMS installed beneath the pin.

The SMS (Fig. 1d) consists of two thin, symmetrical strips with a rectangular cross section. The profile of the SMS is defined by a curve as we use bending as opposed to axial transformation to maximize length change in the SMS without inducing excessive strain. The mechanical behavior of the SMS is parameterized by a cosine curve with amplitude $A$ and thickness $t$. A cosine curve is used to reduce the stress concentration at the ends, as specimens typically fail at the interface between different materials. Figure 1c shows an example of the unit actuator with the bistable mechanism. The stroke length of the bistable mechanism dictates the change in length of the SMS between the fabricated and the activated states. In this design, the stroke length is $20 \mathrm{~mm}$, leading to a $90^{\circ}$ rotation of the trusses. Under this deformation, the maximum strain within the extending and contracting SMS is 0.258 and 0.374 , respectively. While this design can be fabricated in one piece, we choose to separate the bistable mechanism from the SMS so that we are able to investigate each separately.

To achieve temperature-controlled deployment, the glass transition temperatures $T_{\mathrm{g}}$ of the constituting printed materials are obtained using differential scanning calorimetry (DSC). It is found that the $T_{\mathrm{g}}$ of the rigid (Fig. 2a, HiTemp)

FIG. 1. The design of a heat-activated bistable unit actuator. (a) Schematic showing activation of the actuator. The actuator consists of a bistable mechanism and an SMS. The SMS provides the force to activate the bistable mechanism. (b) The design of the unit actuator shows the bistable mechanism consisting of a bracket that provides structural rigidity, joints that provide the rotational DOF to the bistable trusses, and flexible beams that simulate the boundary condition of the von Mises truss. The overall dimensions and the connection points are indicated. The detailed dimensions are shown in Appendix I. (d) The SMS whose geometry is parametrically defined to provide both expansion and contraction to the actuator. (c, e, f) The fabricated specimens. SMS, shape memory strip. 


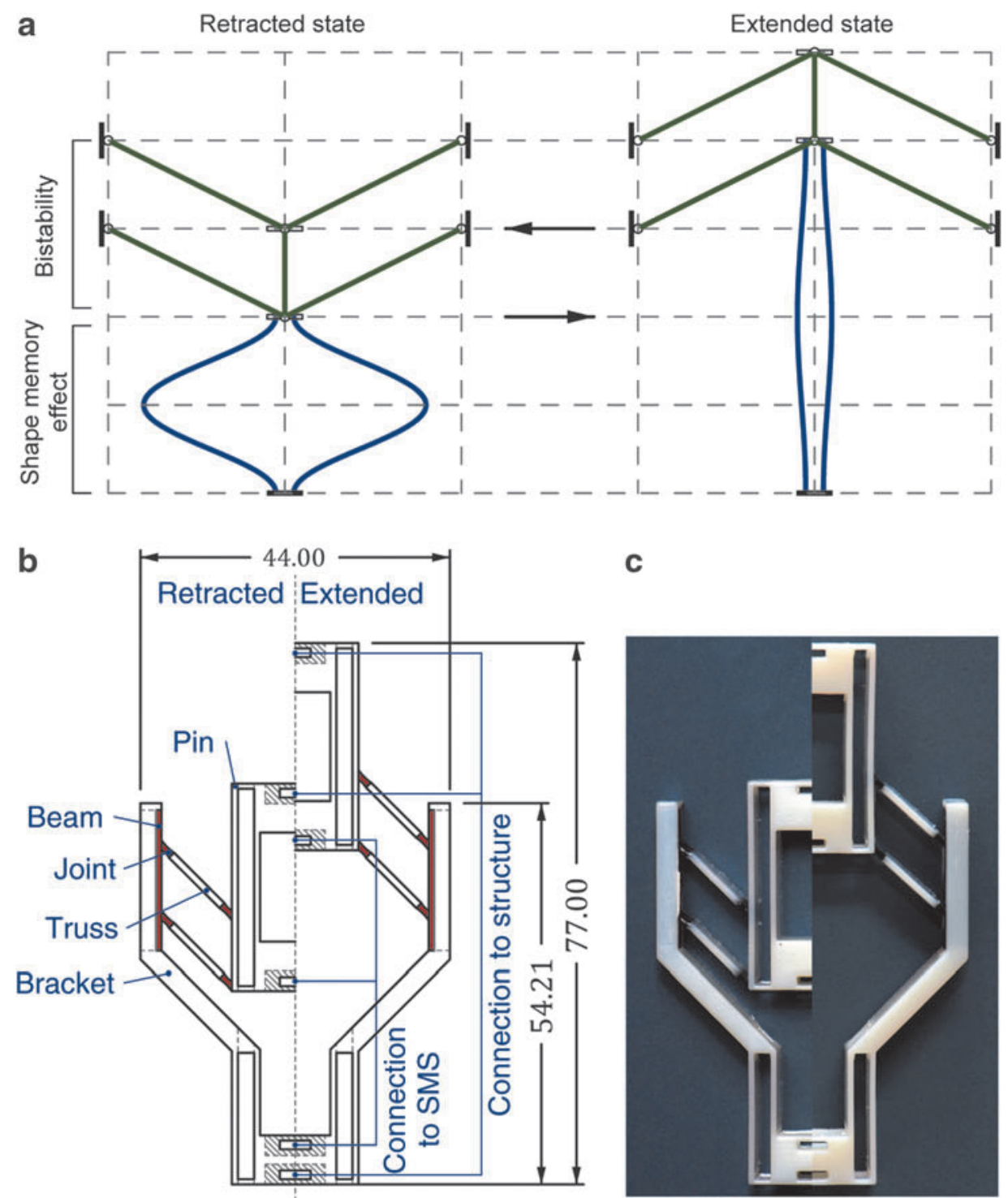

c

Retracted Extended
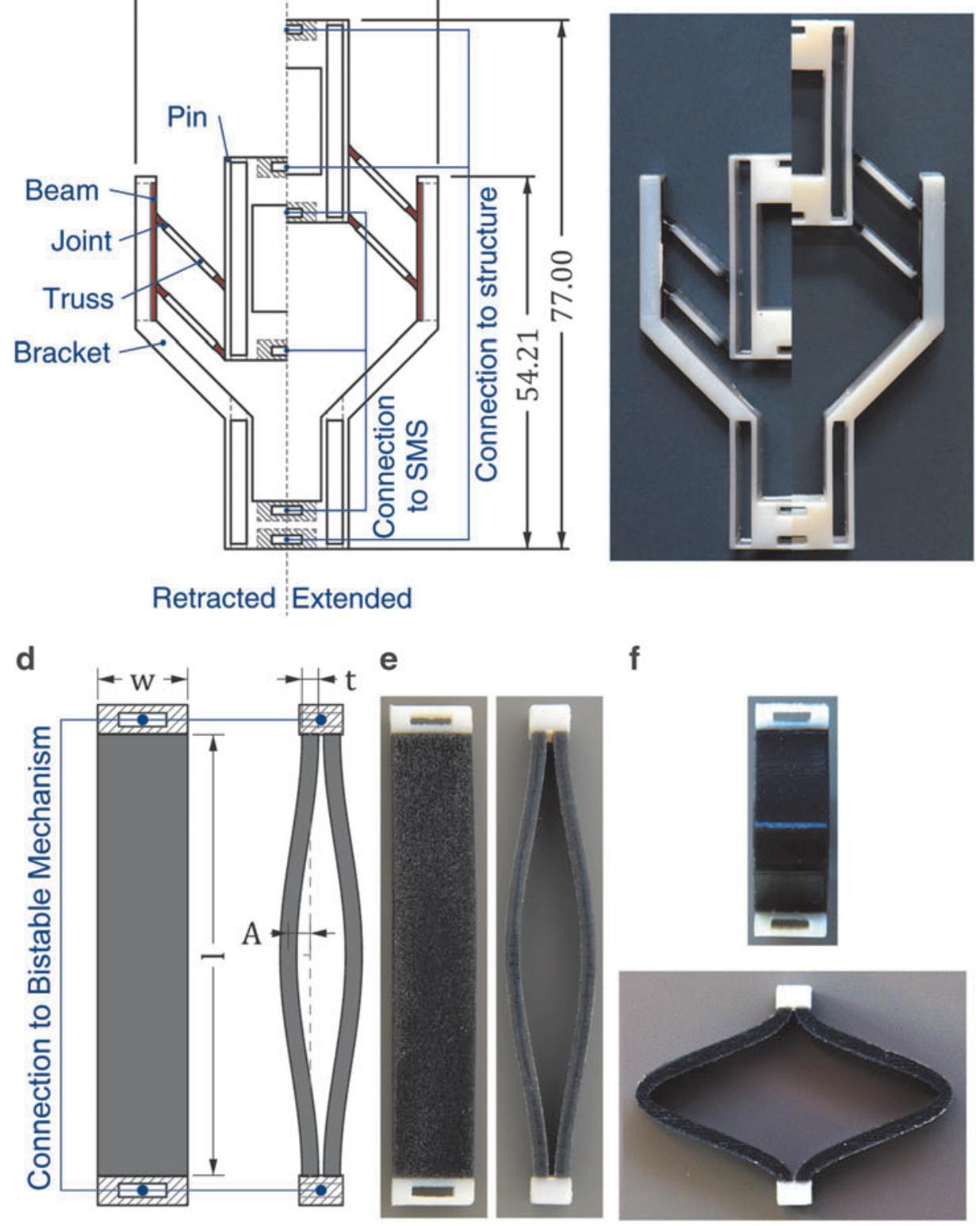

f
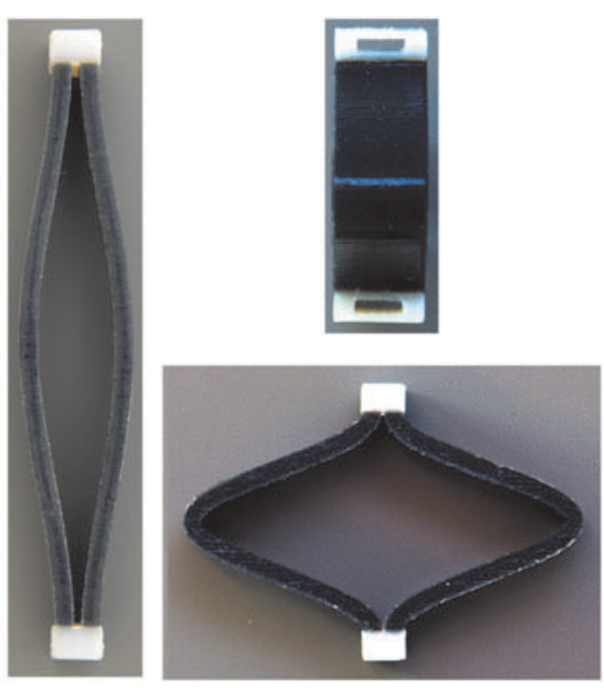

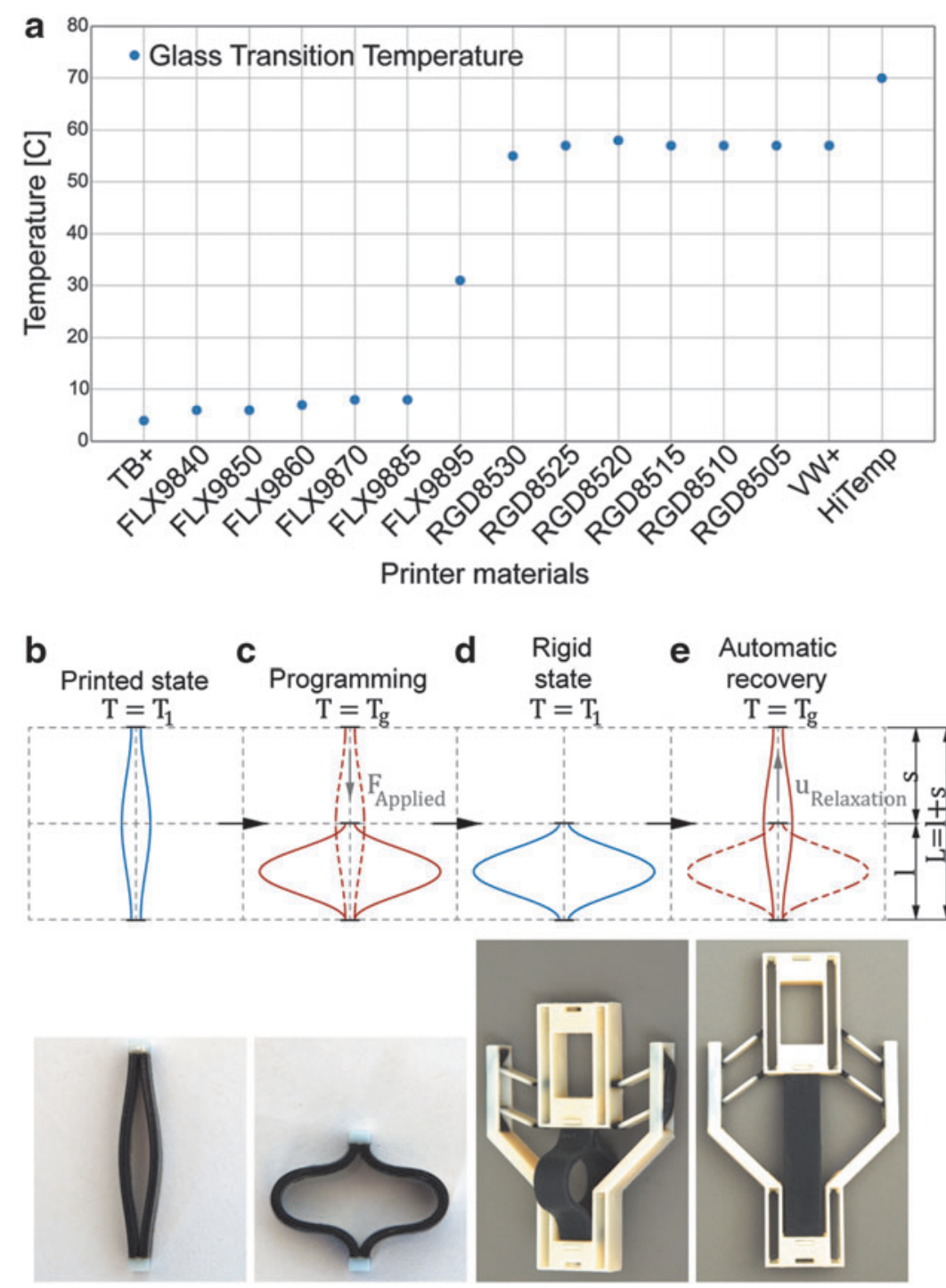

FIG. 2. Glass transition temperature of the different printed materials and the activation sequence of an expanded unit actuator. (a) $T_{\mathrm{g}}$ of the different printed digital materials from differential scanning calorimetry tests. (b) The printed state of an expanded SMS. (c) By heating the SMS past its $T_{\mathrm{g}}$ and applying a mechanical force, the SMS is deformed by the stroke length to its programmed state. (d) The programmed SMS is cooled down and assembled into the bistable mechanism. (e) The unit actuator is activated through heating. On cooling, the final activated state is rigid and behaves as a static member.

and the compliant components (Fig. 2a, Agilus30) are $\sim 70^{\circ} \mathrm{C}$ and $4^{\circ} \mathrm{C}$, respectively. ${ }^{26}$ This effectively renders both thermally stable at a temperature in the vicinity of the $T_{\mathrm{g}}$ of the SMS material, which is $\sim 30^{\circ} \mathrm{C}$ (Fig. 2a, FLX9895). A material with a higher $T_{\mathrm{g}}$ (e.g., Fig. $2 \mathrm{a}$, RGD8530) could also be used for the SMS, however, it was observed with DSC experiments that an increase in heat capacity occurs over a large temperature range, that is, the transition occurs at temperatures within 5 to 10 degrees of the stated $T_{\mathrm{g}}$. The higher activation temperature of RGD8530 can potentially cause the HiTemp material to rubberize as well.

A dynamic mechanical analysis (DMA) is used to obtain the storage modulus of the SMS material. The transformation of the storage modulus to relaxation modulus and modeling of this using the Prony series is detailed in Wagner et al. ${ }^{26}$ The Prony series of the active material is reported in Appendix II.
The deployment of the unit actuator procedure consists of two phases; the first phase is the programming and assembly of the SMS with the bistable mechanism. The second is the constrained recovery of the SMS and consequently the activation of the unit actuator. In the programming phase, the SMS is heated past $T_{\mathrm{g}}$ and is either stretched or compressed by a distance equal to the stroke length of the bistable mechanism (Fig. 2b, c). While confined, the SMS is cooled and installed in the bistable mechanism (Fig. 2d). The second phase is triggered by raising the temperature of the unit actuator past $T_{\mathrm{g}}$ (Fig. 2e). As the SMS recovers, it triggers the bistable mechanism and achieves the deployed state. Once cooled, this activated state behaves as a rigid structure as the SMS returns to its glassy state.

To ensure deployment, the SMS must overcome the activation force of the bistable mechanism at all stages of the deployment process. Mechanical testing is done using a 

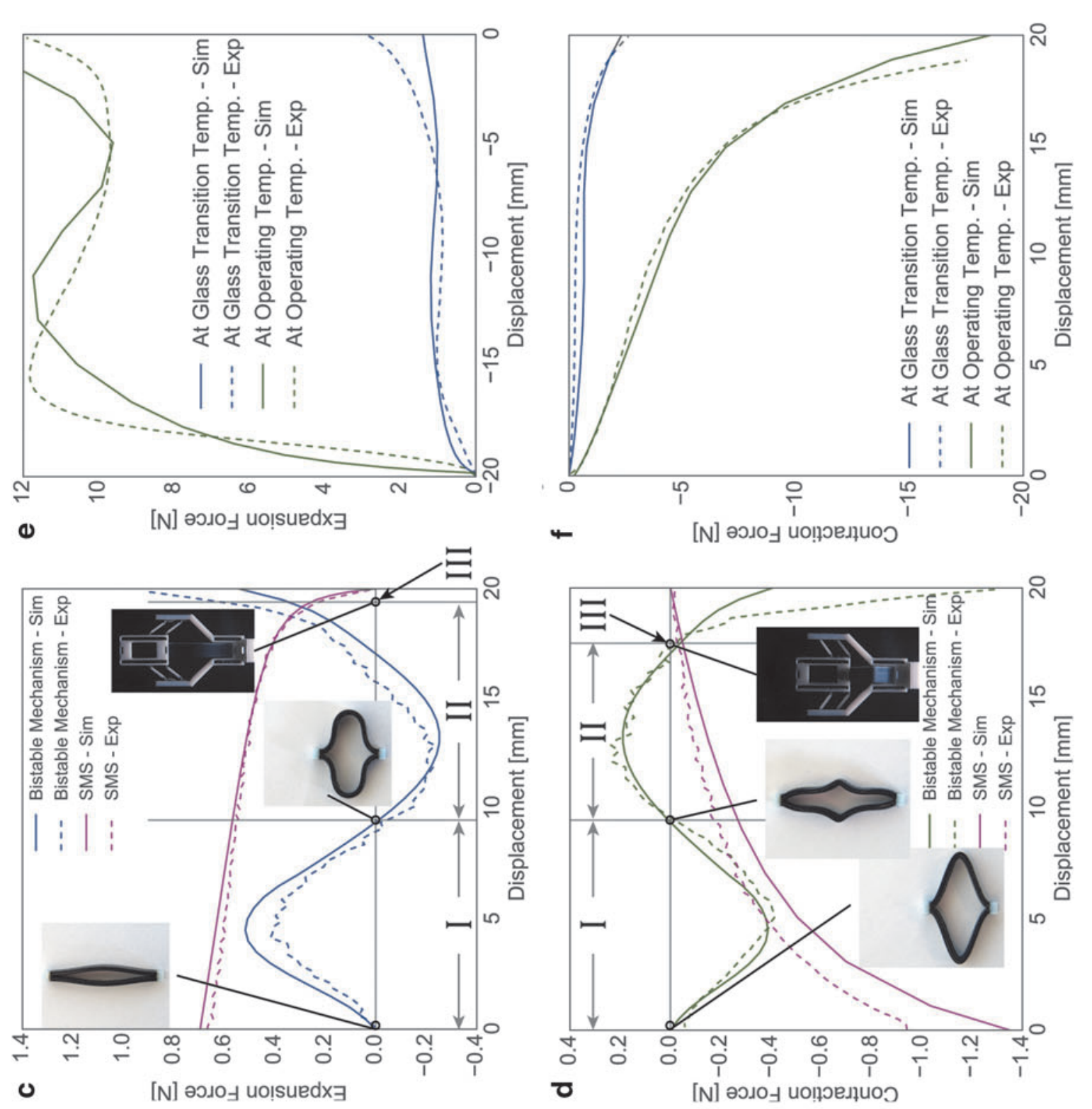

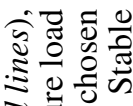

ฐ

氜

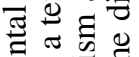

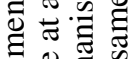

ن

๙

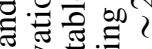

可.

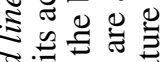

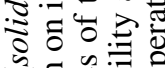

휴 율 है

응

:

क त्रे

응

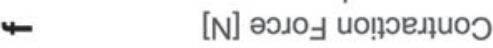

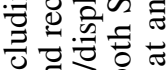

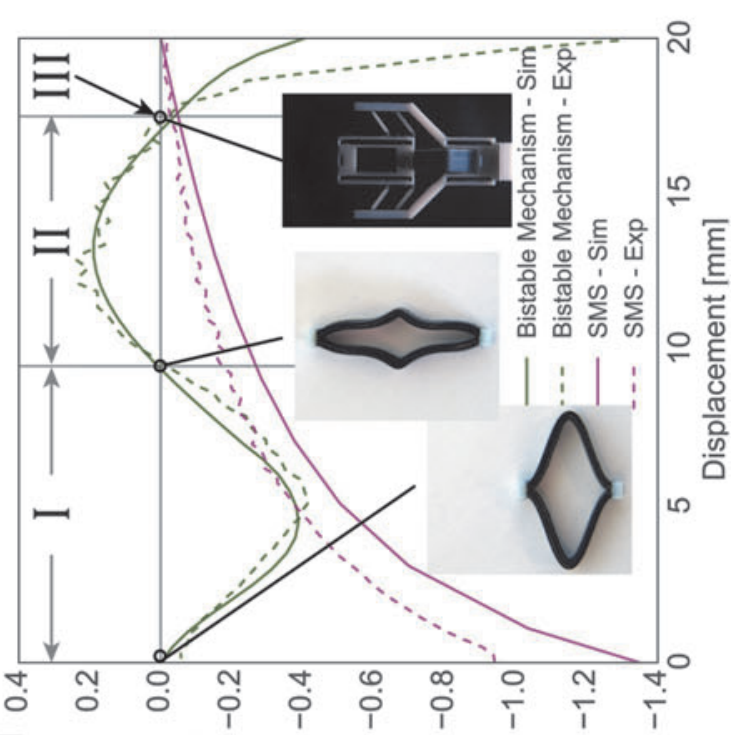

语=

ชే

s.

ติ

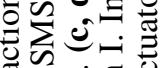

可的它

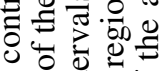

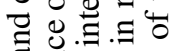

ฮี

。

중

政

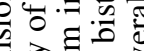

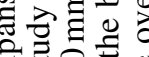

的可

ヨ.

웡

8 저울

踏
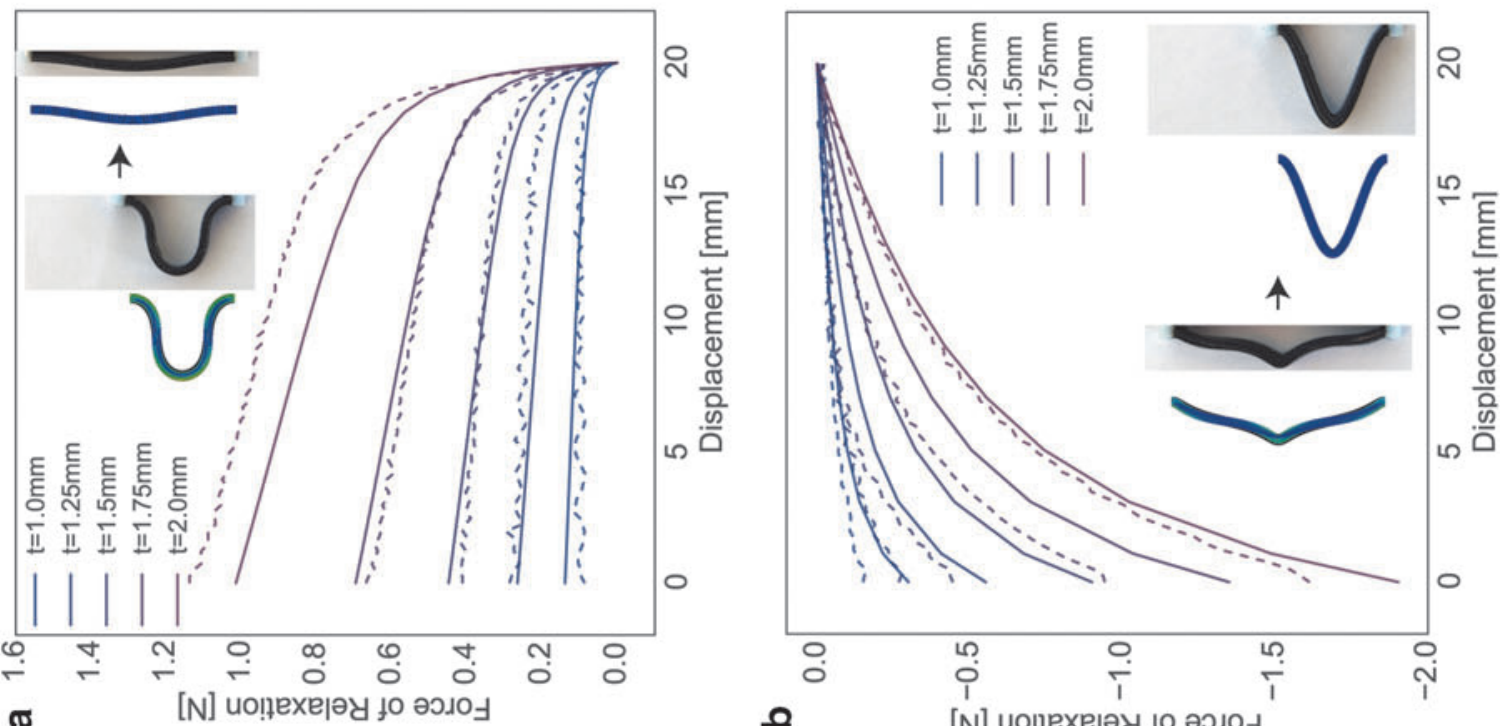

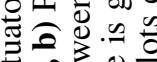

氜过

䒜我远

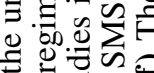

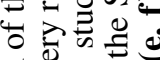

ธี

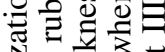

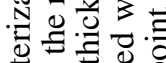

월

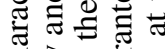

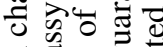

정 $\frac{5}{60} 000$

.

웡

$\sum F$

जo

rio

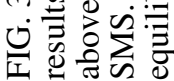



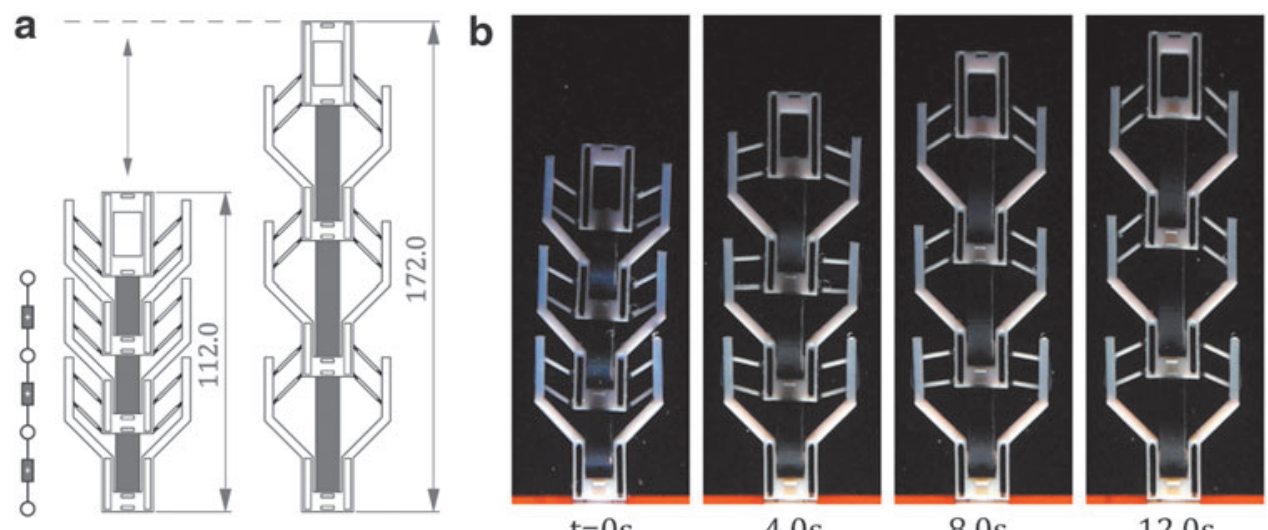

FIG. 4. Sequenced activation of multiple actuators. (a) Schematic showing three expansion actuators connected in series (+) and the physical specimen in both initial and final state. (b) Video screen captures showing the sequenced activation of the actuators. See Supplementary Video S1 (Supplementary Data are available online at www.liebert pub.com/3dp).

dynamic testing machine with an embedded heat chamber. To simulate the test condition, a finite element simulation is performed to obtain the behavior of the SMS using a linear viscoelastic constitutive model constructed for the shape memory material. Static stress and strain behavior of the different materials is obtained from Chen et al. ${ }^{23}$ Simulation of the bistable mechanism follows a static nonlinear FEA with prescribed displacement using beam elements.

Simulation and experimental results are shown in Figure 3. Panels $a$ and $b$ show the force exerted by the SMS during relaxation when $T \geq T_{\mathrm{g}}$. By varying the thickness of the SMS, we are able to tune the force from 0.1 to $1 \mathrm{~N}$ during expansion and 0.1 to $2 \mathrm{~N}$ for contraction. Using these data, we select an SMS with the force required to activate the bistable mechanism. As shown in Panels c and d, to activate the bistable mechanism when $T \geq T_{\mathrm{g}}$, the SMS must deliver a constrained relaxation force that is greater than the bistability activation force, that is, $F_{\mathrm{SMS}}-F_{\mathrm{bi}} \geq 0$. This must be explicitly satisfied in Region I where the bistable force acts in opposition to the SMS. In Region II, after the bistable mechanism triggers, both forces act in the same direction. This enables the force amplification characteristic of the bistable mechanism. Equilibrium occurs at the end of Region II where the two forces balance once again (labeled as III). Note that this is slightly before full stroke length as the bistable mechanism has an asymmetrical behavior.

Panels e and $\mathrm{f}$ show the overall mechanical behavior of a unit actuator under either activation temperature (i.e., $T \geq T_{\mathrm{g}}$ ) or operating temperature (i.e., $T<T_{\mathrm{g}}$, in this case, $T \cong 22^{\circ} \mathrm{C}$ ). In the first instance, the overall behavior equals to the sum of the relaxation force of the SMS and the activation force of the bistable mechanism. In the second instance, by cooling the SMS to its glassy state, its Young's modulus increases significantly. As a result, the unit actuator behaves as if it were a static structural element, allowing a much stiffer force/displacement response.

We demonstrate that the actuator can be integrated into a larger structure by showing the controlled activation of three serially connected actuators. By varying the thickness of the SMS, we can control the sequence of activation due to heat conduction of the shape memory polymer. SMS with thick- nesses of 1.50, 1.75, $2.00 \mathrm{~mm}$ are used as they can all trigger the bistable element, yet have different activation times. The actuator is designed such that its pin is part of the frame of a second, serially connected actuator (Fig. 4a). This increases the overall expansion ratio as more actuators are linked. The activation sequence begins with the thinnest SMS and completes with the thickest SMS (Fig. 4b).

\section{Design of autonomous, multistate shape reconfigurable structures}

We now integrate the described actuators in 2D designs that deploy into load carrying 3D structures. An edge node schematic of the structure is shown in Figure 5a. The design can be simulated as a truss network, that is, the edges can only sustain axial load and the nodes are pin jointed. The design consists of a rectangular frame with four cross edges. Each edge can assume three behaviors: expansion, contraction, or remain static. In this work, two physical realizations of the design are demonstrated by switching the initial configuration of the cross edges between expansion and contraction actuators (Fig. 5b). Compliant joints are placed at the nodes where rotational degrees of freedom (DOF) are required. The stiffness of the joints is reduced by fabricating them with a soft elastomer and internal microstructure. The different parts are assembled with a common joint (Fig. 5c).

Each SMS is programmed separately and assembled into the structure. The initial state is planar. For deployment, the structure is submerged under $40^{\circ} \mathrm{C}$ water. Self-weight of the structures is neglected as the density of the materials is $\sim 1175 \mathrm{~kg} \mathrm{~m}^{-3}$, which is slightly higher than water. Both structures activate as predicted assuming the actuators are springs prestrained by the stroke length. With the expanding structure, a synclastic surface is formed resembling a pyramid. With the contracting structure, an anticlastic surface is formed. The timing of the activation for both is under three minutes, which is significantly faster than using swelling.

In addition to activation, two load cases are tested by placing a specified mass at the apex of each structure. The first is under the deployment condition where the temperature 
a
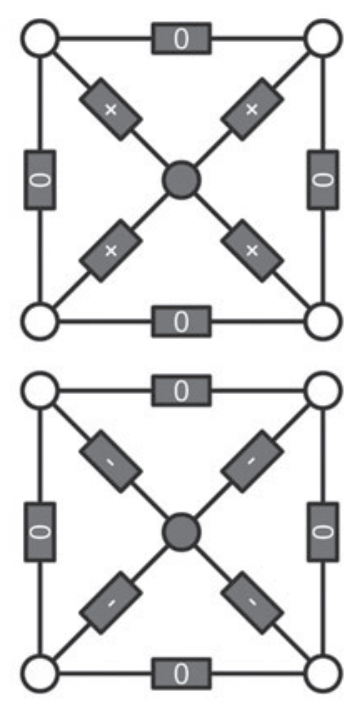

Flat, $\mathrm{T}=\mathrm{T}_{1}$
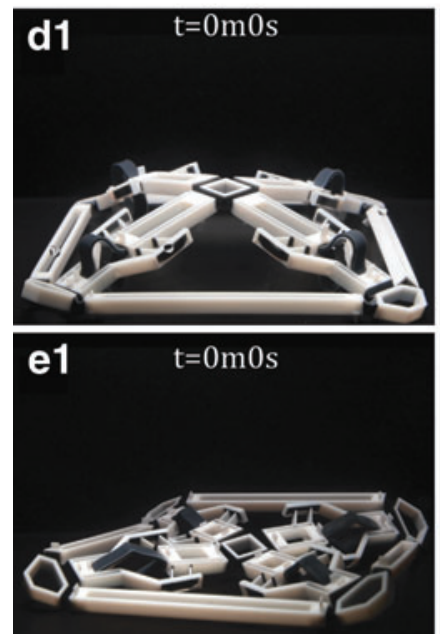

b

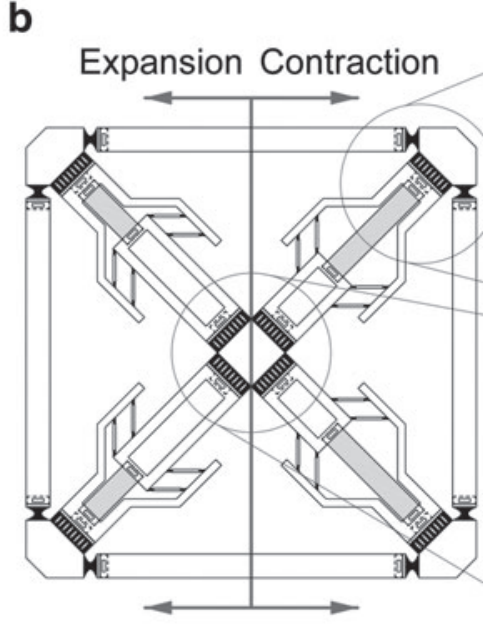

Deployed, $\mathrm{T}=\mathrm{T}_{2}$
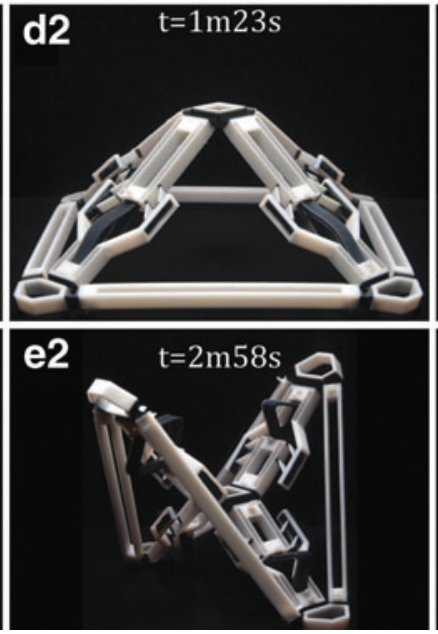

C

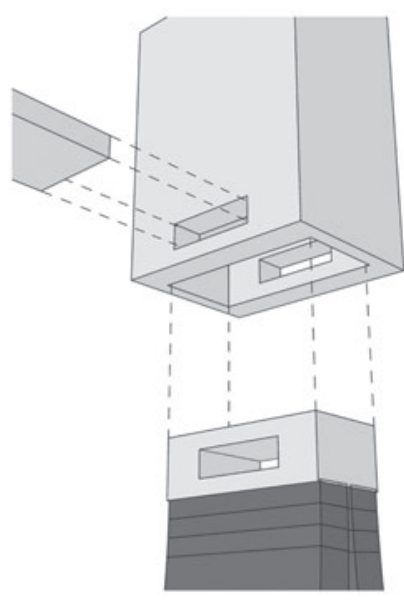

$200 \mathrm{~g}, \mathrm{~T}=\mathrm{T}_{1}$

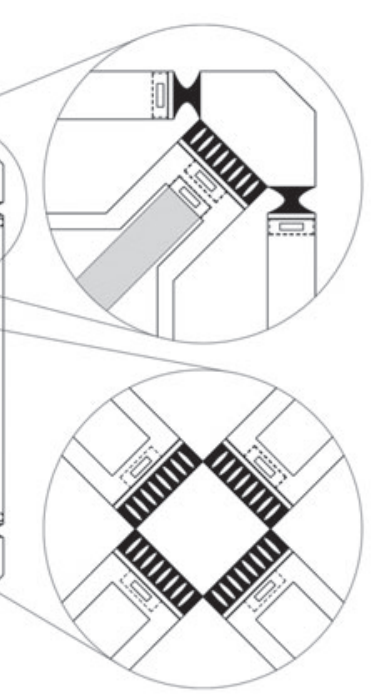

$50 \mathrm{~g}, \mathrm{~T}=\mathrm{T}_{2}$
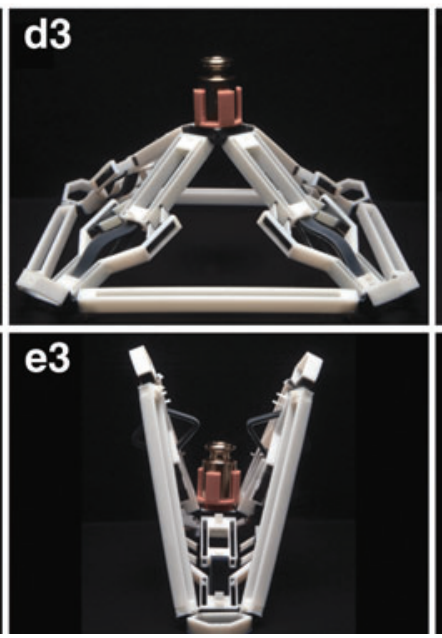
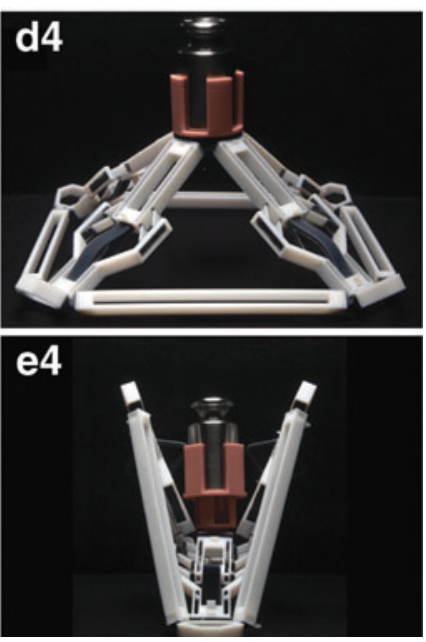

FIG. 5. Shape reconfigurable structures showing activation and load-bearing capability. (a) Schematic of the designs. Four actuators are integrated in a flexible frame, the first has four expansion actuators $(+)$, and the second has four contraction actuators (-). (b) The physical design of the structures, the compliant joints are fabricated with a flexible material and functionally graded to provide the desired flexibility and robustness. (c) A detailed view of a universal connector between all parts of the assembly. (d, e) Video screen captures of the expanding and contracting structural deployment, respectively, (1) flat state, (2) activated state, (3) load-bearing capacity under high temperature, and (4) load-bearing capacity under operating temperature. See Supplementary Videos S2-S7.

remains at $40^{\circ} \mathrm{C}$. The load sustained is $0.050 \mathrm{~kg}$ or an equivalent weight of $0.430 \mathrm{~N}$. The second load case is conducted after the surrounding temperature lowers to $\sim 22^{\circ} \mathrm{C}$. The load sustained is $0.20 \mathrm{~kg}$ or an equivalent weight of $1.70 \mathrm{~N}$.

\section{Materials and Methods}

\section{Fabrication}

All presented designs are fabricated with a Stratasys Objet3 Connex500 multimaterial printer. The three materials used to fabricate the presented designs are FLX9895, the shape memory polymer, RGD525, a temperature-resistant rigid plastic, and Agilus30, an elastomer-like material. ${ }^{27}$ FLX9895 is one of the digital materials created at the time of printing by mixing VeroWhitePlus and Agilus30. SUP760 is used as a chemically dissolvable support material. During postprocessing, most of the support material is removed with manual tools. The remainder is dissolved in a $2 \%$ sodium hydroxide, $1 \%$ sodium silicate solution for $2 \mathrm{~h}$. The specimens are then dipped in an acidic solution and air-dried before testing.

\section{Experimental}

Mechanical characterization of the SMS is performed in an Instron E3000 dynamic testing machine with an installed thermal chamber. The specimen is clamped with grippers. The interior volume of the heat chamber is heated to $40^{\circ} \mathrm{C}$. This temperature is maintained for $30 \mathrm{~min}$ before testing to 


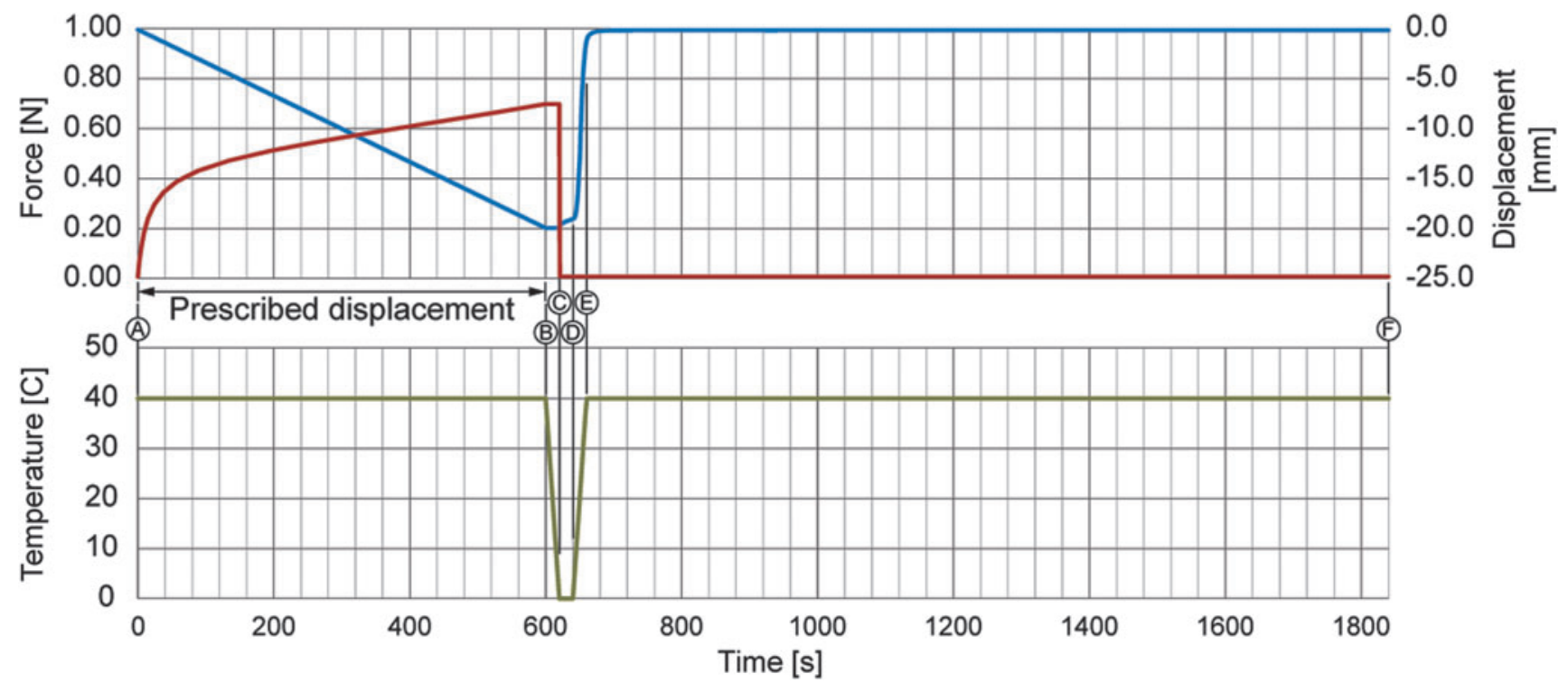

FIG. 6. Simulation of the SMS with viscoelastic brick elements during programming and relaxation. From A to B, a displacement is prescribed under $\mathrm{T}=T_{\mathrm{g}}$, the reaction force is the output. From B to $\mathrm{C}$, the prescribed displacement continues to be applied as the temperature decreases to $0^{\circ} \mathrm{C}$. From $\mathrm{C}$ to $\mathrm{D}$, the displacement is removed, a slight rebound is observed in deformation. From D to E, temperature increases and relaxation occurs. This continues to F.

ensure thermal equilibrium is reached. A displacement of $20 \mathrm{~mm}$ is applied and removed at a rate of $0.05 \mathrm{~mm} \mathrm{~s}^{-1}$. The reaction force is recorded with a load cell. This displacement is tensile for the extending SMS and is compressive for the contracting SMS, and the magnitude of the displacement equals the stroke length of the bistable mechanism. This experiment provides the stress/strain behavior of the material under the rubbery state and ensures that the resulting recovery force is sufficient to overcome the bistable mechanism's activation force.

Testing of the deployable structures is done under water that is heated with a heating element. For deployment, water is heated past $T_{\mathrm{g}}$ before the specimens are submerged. Due to the fast activation time, the specimens are slightly activated by the time they are placed at the base of the water tank. The water is cooled below $T_{\mathrm{g}}$ to test structural behavior under operating conditions.

\section{Simulation}

Numerical simulation of the unit actuator is done using the finite element analysis software ABAQUS 6.14.1. The bistable mechanism is meshed with a nonlinear beam element B32 to reduce computational effort compared with brick elements while retaining accuracy. The flexible beam is assumed to be clamped on the ends. A hyperelastic material model is used for Agilus30 (data shown in Appendix II). A displacement of $20 \mathrm{~mm}$ is prescribed to avoid snap through and obtain the portion of the stress/strain curve with negative stiffness.

Simulation of the SMS consists of a multistep analysis (Fig. 6). The SMS is meshed with viscoelastic brick elements C3D8RH. A displacement of $20 \mathrm{~mm}$ is incrementally applied under the heated condition (Step A to B). This displacement is maintained while the temperature cools to $0^{\circ} \mathrm{C}$ (Step B to
C) and is removed from Step C to D. A slight rebound in displacement is observed. From Step D to E, the temperature is raised to $40^{\circ} \mathrm{C}$ and the relaxation is observed. Relaxation continues after the temperature reaches steady state.

\section{Discussion and Conclusions}

The presented work demonstrates the design of autonomous shape reconfigurable structures. We propose a programmable unit actuator that either expands or contracts under temperature change. Using the viscoelastic behavior of thermosetting polymers, a prestrain is imposed on the SMS at a temperature greater than the polymer's glass transition temperature, $T_{\mathrm{g}}$. This prestrain locks in when the temperature lowers and is released when the temperature increases again, thereby triggering the bistable mechanism. Using this actuator, we demonstrate the deployment of 2D sequenced actuators and load-bearing 3D space structures.

The proposed unit actuator can be integrated in structural applications where the transport volume must be much smaller than the operating volume. With respect to the example of deployable space structures, the proposed design has a number of advantages compared with the state-of-art in all phases of the deployment process.

In the fabrication phase, both the bistable mechanism and the SMS are 3D printed with minimal support material and assembled with a universal connector (Fig. 5c). The activation force of the bistable mechanism is dependent on its joint length and stiffness. ${ }^{23}$ The triggering temperature and duration of the SMS can be tuned by varying the chemical composition of the shape memory polymer resin ${ }^{19}$ and by changing its thickness, respectively.

In transportation and the deployment phase, the stowed or programmed state of the proposed structure is precisely defined by the first equilibrium point of the bistable mechanism. 
This stowed state is maintained without a restraining mechanism. ${ }^{28}$ Rather than motorized deployment, the proposed structures are activated through environmental change, thereby removing the need for electrical energy input and eliminating the possibility of jamming of parts.

While we demonstrate initially flat structures as an example, one is able to design the stowed state to accommodate given space requirements. By contrast, designs that feature only SMPs are programmed with limited precision using $a d$ hoc methods such as hand pulling. ${ }^{29}$ The proposed structures are load bearing as they are deployed, that is, under high temperature, as the bistable mechanism amplifies the loadbearing capacity of the unit actuator independently of the surrounding temperature. This is in contrast with most 4Dprinted designs that focus solely on shape transformation ${ }^{8}$ and do not exhibit significant load-bearing capacity during deployment but rather only after the deployed design cools below $T_{\mathrm{g}}$. Under operating temperature (i.e., $T<T_{\mathrm{g}}$ ), the SMS returns to its glassy state and behaves as a rigid member, effectively making the unit actuator a static structural element.

In addition to autonomous deployment, the combination of the shape memory effect and mechanical bistability may have broad relevance in other engineering fields. A recent study by the same authors has shown a directional swimming robot propelled by exploiting the instability of a bistable mechanism triggered with a shape memory polymer. ${ }^{30}$ As demonstrated, the structures can reconfigure themselves both positively and negatively, with a combination of these two modes and given many actuators, one can form a surface of almost any given curvature. As we have characterized the thermal mechanical behavior of the unit actuator, simulation of the deployment and load capacity can be done using formfinding algorithms, developed in previous work, that can be extended to generate and optimize large-scale reconfigurable structures. $^{23}$

\section{Acknowledgments}

The authors are grateful to Jung-Chew Tse from the Engineering Design and Computing Laboratory, ETHZ, for fabrication support, Marius Wagner from the Laboratory for Nanometallurgy, ETHZ, for performing the DMA and data analysis, and Dr. Joanna Wong from the Laboratory of Composite Material and Adaptive Structures, ETHZ, for performing DSC experiments.

\section{Author Disclosure Statement}

No competing financial interests exist.

\section{References}

1. Lan X, Liu Y, Lv H, Wang X, Leng J, Du S. Fiber reinforced shape-memory polymer composite and its application in a deployable hinge. Smart Mater Struct 2009;18: 24002.

2. Leong TG, Randall CL, Benson BR, Bassik N, Stern GM, Gracias DH. Tetherless thermobiochemically actuated microgrippers. Proc Natl Acad Sci U S A 2009;106: 703-708.

3. Guo X, Li H, Ahn BY, et al. Two- and three-dimensional folding of thin film single-crystalline silicon for photovoltaic power applications. Proc Natl Acad Sci U S A 2009; 106:20149-20154.

4. Wang Q, Gossweiler GR, Craig SL, Zhao X. Cephalopodinspired design of electro-mechano-chemically responsive elastomers for on-demand fluorescent patterning. Nat Commun 2014;5:4899.

5. Ryu J, D'Amato M, Cui X, Long KN, Jerry Qi H, Dunn ML. Photo-origami-bending and folding polymers with light. Appl Phys Lett 2012;100;161908.

6. Maccurdy R, Katzschmann R, Kim Y, Rus D. Printable hydraulics: A method for fabricating robots by $3 \mathrm{D}$ co-printing solids and liquids. In: Proceedings of the 2016 IEEE International Conference on Robotics and Automation (ICRA), Stockholm, Sweden, 2016; pp. 3878-3885.

7. Ge Q, Qi HJ, Dunn ML. Active materials by four-dimension printing. Appl Phys Lett 2013;103;131901.

8. Tibbits S. 4D printing: multi-material shape change. Archit Des High Defin Zero Toler Des Prod 2014;84: 116-121.

9. Song YS, Sitti M. Surface-tension-driven biologically inspired water strider robots: Theory and experiments. IEEE Trans Robot 2007;23:578-589.

10. Zolesi VS, Ganga PL, Scolamiero L, et al. On an innovative deployment concept for large space structures. In: 42nd International Conference on Environmental Systems, San Diego, CA, 2012; p. 3601.

11. Gärdsback M, Tibert G, Izzo D. Design considerations and deployment simulations of spinning space webs. In: 48th AIAA/ASME/ASCE/AHS/ASC Structures, Structural Dynamics, and Materials Conference, Norfolk, VA, 2007; p. 1829.

12. Mitsugi JIN, Ando K, Senbokuya Y, Meguro A. Deployment analysis of large space antenna using flexible multibody dynamics simulation. Acta Astronaut 2000;47: 19-26.

13. Miura K. Method of packaging and deployment of large membranes in space. Inst Space Astronaut Sci Rep 1985; 618:1-9.

14. Liu K, Wu J, Paulino GH, Qi HJ. Programmable deployment of tensegrity structures by stimulus-responsive polymers. Sci Rep 2017;7:3511.

15. Clem A, Smith S, Main J. A pressurized deployment model for inflatable space structures. In: 41st Structures, Structural Dynamics, and Materials Conference and Exhibit, Atlanta, GA, 2000; p. 1808.

16. Edwards BC. Design and deployment of a space elevator. Acta Astronaut 2000;47:735-744.

17. Raviv D, Zhao W, McKnelly C, et al. Active printed materials for complex self-evolving deformations. Sci Rep 2014;4:7422.

18. Sydney Gladman A, Matsumoto EA, Nuzzo RG, Mahadevan L, Lewis JA. Biomimetic 4D printing. Nat Mater 2016;15:413-418.

19. Ge Q, Sakhaei AH, Lee H, Dunn CK, Fang NX, Dunn ML. Multimaterial 4D Printing with tailorable shape memory polymers. Sci Rep 2016;6:31110.

20. Ding Z, Yuan C, Peng X, Wang T, Qi HJ, Dunn ML. Direct 4D printing via active composite materials. Sci Adv 2017; 3:e1602890.

21. Schioler T, Pellegrino S. Space frames with multiple stable configurations. AIAA J 2007;45:1740-1747.

22. Shan S, Kang SH, Raney JR, et al. Multistable architected materials for trapping elastic strain energy. Adv Mater 2015;27:4296-4301. 
23. Chen T, Mueller J, Shea K. Integrated design and simulation of tunable, multi-state structures fabricated monolithically with multi-material 3D printing. Sci Rep 2017;7: 45671.

24. Strauss S, Krog RL, Feiveson AH. Extravehicular mobility unit training and astronaut injuries. Aviat Space Environ Med 2005;76:469-474.

25. Cedolin L. Stability of structures: Elastic, inelastic, fracture and damage theories. Signapore: World Scientific, 2010.

26. Wagner M, Chen T, Shea K. Large shape transforming 4D auxetic structures. 3D Print Addit Manuf 2017;4:133-142.

27. Stratasys Ltd. Materials; 2018. www.stratasys.com/materials/ search Accessed May 3, 2018.

28. Brinkmeyer A, Pellegrino S, Weaver PM. Effects of longterm stowage on the deployment of bistable tape springs. J Appl Mech 2015;83:11008.

\section{Appendix}

\section{Appendix I: Detailed Design of the Structures}

While the actuator can be fabricated monolithically, it is partitioned into two parts, the bistable mechanism and the SMS, to drastically reduce printing cost and time. The bistable mechanism consists of two pairs of $45^{\circ}$ inclined trusses. The spacing between the truss pairs is $10 \mathrm{~mm}$, resulting in a stroke length of $20 \mathrm{~mm}$ (Appendix Fig. A1). The SMS profile follows a cosine curve, $y(x)=A \cos (x+\pi), 0<x<L$. For the expanding SMS, $A=2.00 \mathrm{~mm}$ and $L=40.5 \mathrm{~mm}$, and for
29. Ge Q, Dunn CK, Qi HJ, Dunn ML. Active origami by 4D printing. Smart Mater Struct 2014;23:1-15.

30. Chen T, Bilal OR, Shea K, Daraio C. Harnessing bistability for directional propulsion of untethered, soft robots. Proc Natl Acad Sci [Epub ahead of print]; DOI: 10.1073/pnas .1800386115 .

Address correspondence to:

Kristina Shea

Engineering Design and Computing Laboratory

Department of Mechanical and Process Engineering Swiss Federal Institute of Technology Zurich

Tannenstrasse 3, CLA F35

Zurich 8092

Switzerland

E-mail: kshea@ethz.ch

the contracting SMS, $A=16.8 \mathrm{~mm}$ and $L=20.5 \mathrm{~mm}$. The amplitude of the contracting SMS is set such that the resulting curve length of the two SMS is identical. The thickness of the SMS is varied to deliver the required recovery force.

The deployable structures presented in the article, Figure 5, are $169.71 \times 169.71 \mathrm{~mm}$ in the enveloping dimension. Since the design requires pin-jointed connections between the edges, the embedded actuators (Appendix Fig. A1) are connected to each other and to the static members using architected compliant joints (Appendix Fig. A2).

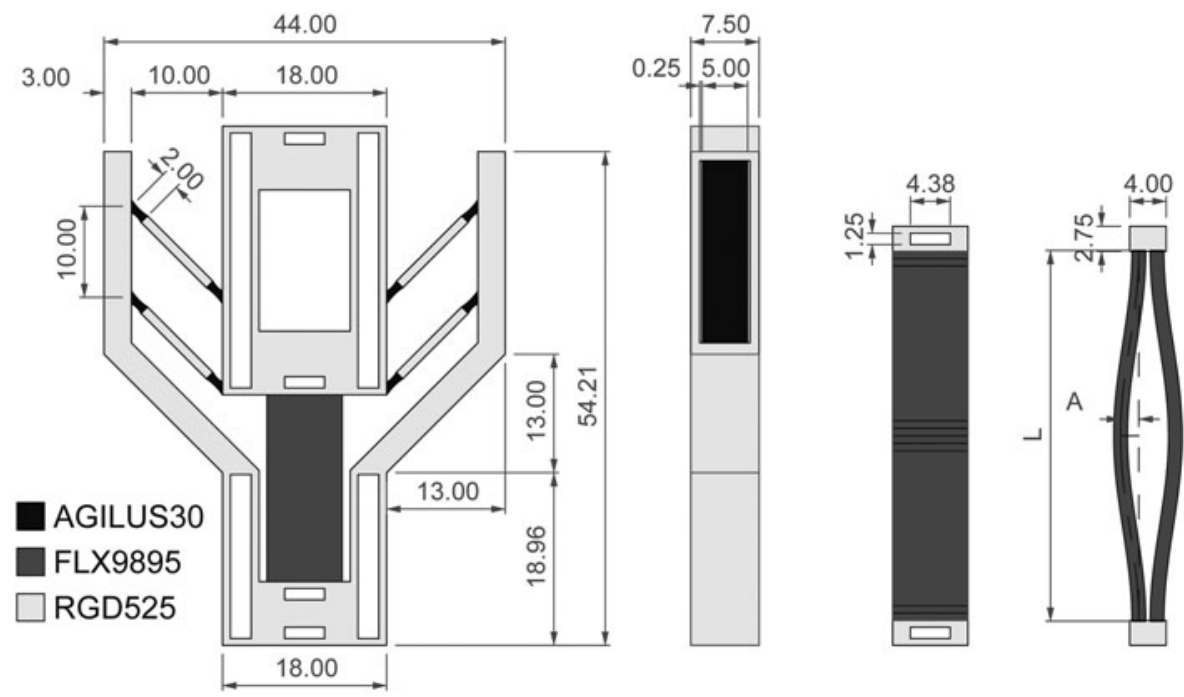

APPENDIX FIG. A1. Designs of the bistable mechanism and the SMS. The bistable mechanism features two pairs of inclined trusses angled at $45^{\circ}$, resulting in a stroke length of $20 \mathrm{~mm}$. The SMS follows a parametric profile of a cosine curve. SMS, shape memory strip. 

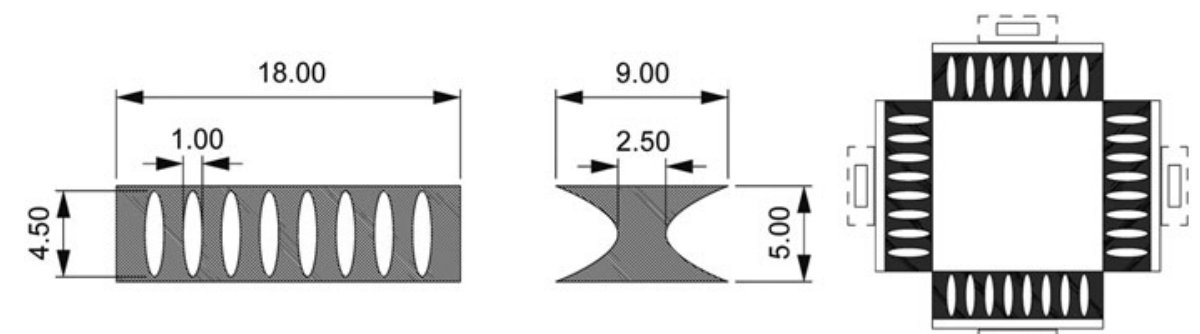

노

APPENDIX FIG. A2. Designs of the architected compliant joints. They provide a rotational DOF perpendicular to the two joined members but restrain the DOFs in the other directions. By using multiple compliant joints, we approximate an idealized pin joint. DOF, degree of freedom.

\section{Appendix II: Material Characterization Data}

Appendix Table A1. Hyperelastic Material Data of Agilus30, Using Polynomial Fit $\mathrm{N}=2$

\begin{tabular}{ccccccc}
\hline $\mathrm{C}_{10}$ & $\mathrm{C}_{01}$ & $\mathrm{C}_{20}$ & $\mathrm{C}_{11}$ & $\mathrm{C}_{02}$ & $\mathrm{D}_{1}$ & $\mathrm{D}_{2}$ \\
\hline-0.4886 & 0.7147 & 0.07929 & -0.2704 & 0.4709 & 0.4574 & 0.0 \\
\hline
\end{tabular}

Appendix Table A2. Prony Series Data of FLX9895

\begin{tabular}{lccc}
\hline$\tau_{\mathrm{i}}$ & $\mathrm{G}_{\mathrm{i}}$ & $\tau_{\mathrm{i}}$ (cont.) & $\mathrm{G}_{\mathrm{i}}$ (cont.) \\
\hline $2.0 \mathrm{E}-09$ & 0.037155 & $1.0 \mathrm{E}+00$ & 0.023205 \\
$1.0 \mathrm{E}-08$ & 0.036325 & $5.0 \mathrm{E}+00$ & 0.038059 \\
$5.0 \mathrm{E}-08$ & 0.019428 & $1.0 \mathrm{E}+01$ & 0.004878 \\
$1.0 \mathrm{E}-07$ & 0.036963 & $5.0 \mathrm{E}+01$ & 0.015653 \\
$5.0 \mathrm{E}-07$ & 0.025824 & $1.0 \mathrm{E}+02$ & $5.27 \mathrm{E}-14$ \\
$1.0 \mathrm{E}-06$ & 0.048254 & $5.0 \mathrm{E}+02$ & 0.003613 \\
$5.0 \mathrm{E}-06$ & 0.029066 & $1.0 \mathrm{E}+03$ & 0.000806 \\
$1.0 \mathrm{E}-05$ & 0.061186 & $5.0 \mathrm{E}+03$ & 0.001064 \\
$5.0 \mathrm{E}-05$ & 0.047911 & $1.0 \mathrm{E}+04$ & 0.000212 \\
$1.0 \mathrm{E}-04$ & 0.048443 & $5.0 \mathrm{E}+04$ & 0.00029 \\
$5.0 \mathrm{E}-04$ & 0.046758 & $1.0 \mathrm{E}+05$ & 0.000218 \\
$1.0 \mathrm{E}-03$ & 0.081033 & $1.0 \mathrm{E}+06$ & 0.000239 \\
$5.0 \mathrm{E}-03$ & 0.088524 & & \\
$1.0 \mathrm{E}-02$ & 0.069187 & & \\
$5.0 \mathrm{E}-02$ & 0.11156 & & \\
$1.0 \mathrm{E}-01$ & 0.043557 & & \\
$5.0 \mathrm{E}-01$ & 0.079339 & & \\
\hline
\end{tabular}

Appendix Table A3. Williams-Landel-Ferry Shift FunCTION CONSTANTS

\begin{tabular}{lcc}
\hline$\theta_{0}$ & $\mathrm{C}_{1}$ & $\mathrm{C}_{2}$ \\
\hline 275 & 19.982 & 109.53 \\
\hline
\end{tabular}

